\title{
Estenosis intestinal postenterocolitis necronizante
}

\author{
Ures. Adolfo Dolz A.,* Alfredo del Río M.**
}

\begin{abstract}

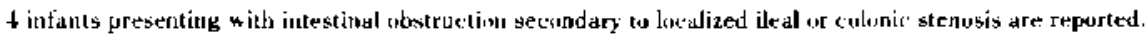

The patients were sperated in the pariud 1976-1979 and the reapective listaries suggeated previsus episuxtes al necratizing enteruculitis in every chase.

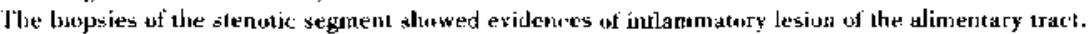

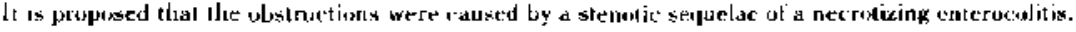

La enterucolitis necronizante del R.N. (E.N.), descrita en forma completa en 1963 por Waldhausen, ${ }^{14}$ es una enfermedad grave, que puede adquirir un tratamiento quirurgico en el período agudo de su evolución. 110.11 .131

Krasna ${ }^{(6)}$ en 1970, fue el primero en describir casus de estenosis intestinales localizadas, adquiridas, como secuelas de enterocolitis necronizante.

La E.N. en la literatura aparece con más frecuencia en la edad de recién nacido, en prematuros con signos de sepsis y que han presentado stress perinatal Su etiopatogenia se relaciona básicamente en orden de importancia con:

1." Isquemia Intestinal. Esta teoría, propuesta por Lloyd ${ }^{\text {(8) }}$ manitiesta que la hipoxia en el recién nacido, por mecanismo neurugénico, produce una disminución del flujo sanguíneo en el intestino para mantener una perfusión adecuada a nivel cerebral y coronario, similar a lo que ocurre en los mamiferos acuáticos. La disminución del riego sanguíneo intestinal por vasoconstricción podría ucasionar necrosis isquémica de la mucosa. ${ }^{\{12)}$ Esto puede ocurrir también durante episodjos de hipotermia o cateterismo umbilical, principalmente en la exanguineotransfusión.

2." Deficiencia Imnunológica. La inmunoglobulina $\mathrm{A}$ está ausente en la superficie de la mucosa intestinal del R.N. como consecuencia de la falta de estimulo antigénico en la vida intrauterina. Una vez que comienza la colonización bacteriana, después del nacimiento, la l.g.A. demora 4 a 6 semanas en alcanzar niveles normales, y más aún en los prematuros. El calostro materno tiene altas concentraciones de I.g.A. y es la fuente d.adora que suple esta carencia en esta etapa de la vida. Por ello, la alimen-

\footnotetext{
"Servicios de Cirugia Infantil Hospital L. Calvo Mackensa. *"Jete de Cirugia Intantsl Hospital L. Lalvo Mackena.
}

tación artificial se constituye en factor predisponente a la enterocolitis necronizante. ${ }^{(i)}$

3." Las dietas hiperosmolares pueden causar, además, lesión directa en la mucosa intestinal.

4." La colonización bacteriana es más discutida y tendría importancia solamente en presencia de los factores anteriores.

La etiopatogenia se resume en el esquema de Burringtón. ${ }^{2 \prime}$

En nuestro medic la E.N. además de presentarse en R.N., aparece con similar frecuencia en laclantes de uno a seis meses, generalmente desnutridos, y con episodios de diarrea aguda con deshidratacion $y$ acidosis."

Esta grave enfermedad con diagnóstico precoz y tratamiento adecuado ha superado su altisima mortalidad hasta alcanzar, en algunas publicaciones, ${ }^{(2,4)}$ sobrevidas de 70 y $80 \%$. Esto reviste especial importancia, por la creciente incidencia de esta patología, que en algunos centros de R.N. ${ }^{(2)}$ de alto riesgo se ha alzado de $2,5 \%$ a $15 \%$.

El diagnóstico se suspecha en prematuros con hipoxia por distress respiratorio o cardiaco. Se agrega sepsis y aparecen letargia, inestabilidad térmica, períodos de apnea y acidosis. Posteriormente vómitos biliosos, distensión abdominal y diarrea con sangre o melena.

Ayuda al diaznóstico la radiografía de abdomen simple, repetida cada 12 horas, que revela distensión de asas 0 , más especificamente, gas intramural.

El hemograma presenta leucocitosis, desviación a la izquierda y trombocitopenia.

El tratamiento adecuado y precoz consiste en:

- Aspiración nasogástrica.

- Alimentación parenteral.

- Corrección hidroelectrolítica y ácido base.

- Antibioterapia. Penicilina y gentamicina o amikacina. 


\section{ESQUEMA ENTEROCOLITIS NECROTIZANTE}

(Burrington)

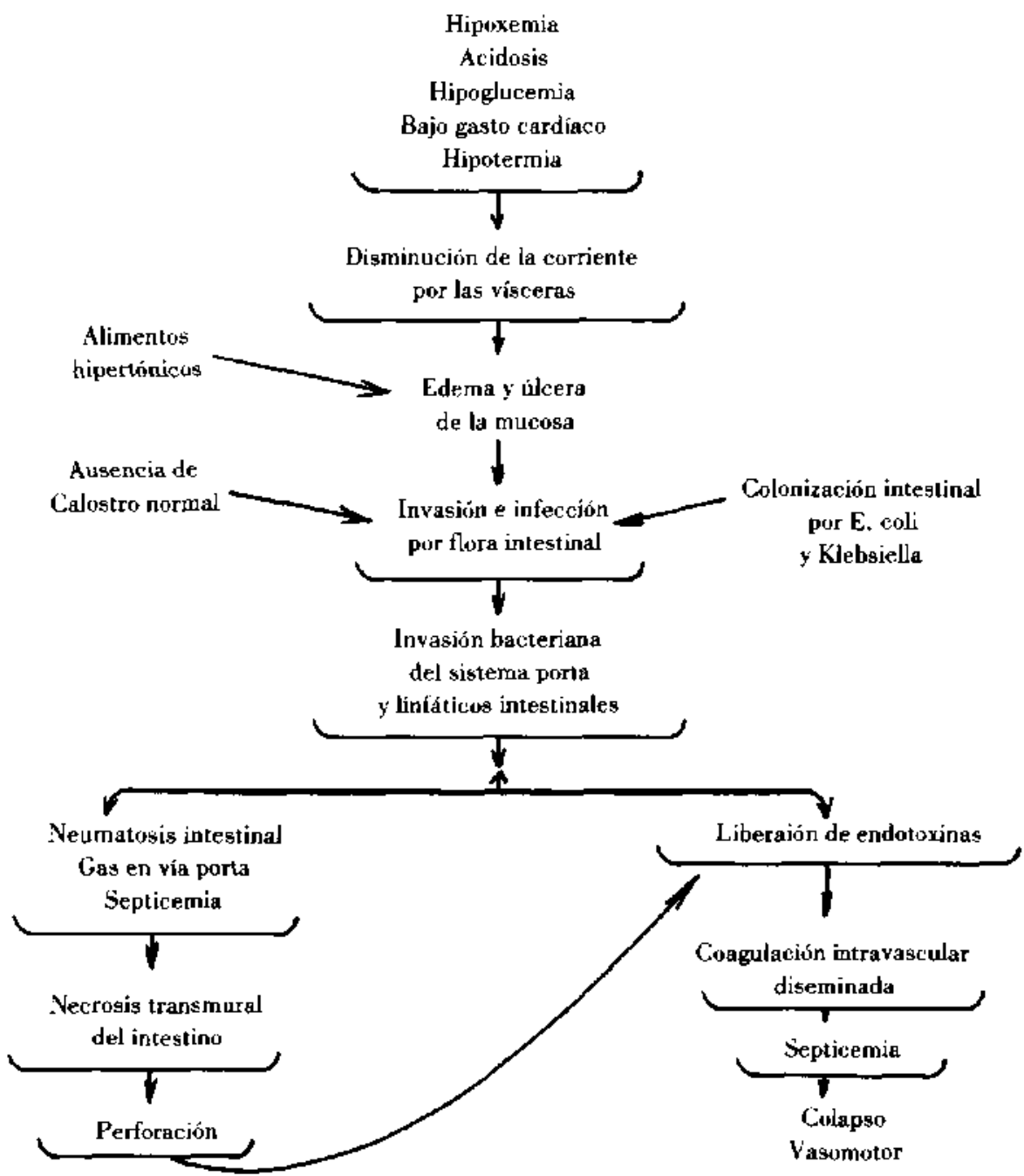

Algunos autore $x^{\text {th }}$ recomiendan instilación de antibióticos locales por sonda nasogástrica y Dextran intravenosu.

Las indicaciones quirúrgicas's. 11' están dadas por signos de peritonitis o perforación:

- Neumoperitoneo.

- Defensa muscular.

- Masa palpable.

- Celulitis de la pared abdominal con enrojecimiento y edema.

- Agravamiento progresivo con tendencia al shock, a pesar del manejo médico adecuado.

- En la radiogratía abdominal simple, presencia permanente de un asa dilatada durante dos o más controles.
El tratamiento quirúrgico consiste en resección de todas las zonas francamente necróticas y una 0 más ostomías, según sea necesario. Cuando la resección es masiva, debe tratarse de preservar la válvula ileocecal.

Las lesiones se distribuyen en forma diseminada a lo largo del intestino, con zonas de necrosis de variable magnitud, o bien pueden estar localizadas en segmentos o focos únicos, más trecuentes en ileon y colon.

A reces el daño se presenta con ulceraciones de la mucosa y edema y hemorragia que comprometen sucesivamente mucosa. submucosa y muscular. De esto se desprende que una alteración localizada pueda pasar inadvertida en el episodio agudo, y más tarde ser rotulada de estenosis idiopática. 


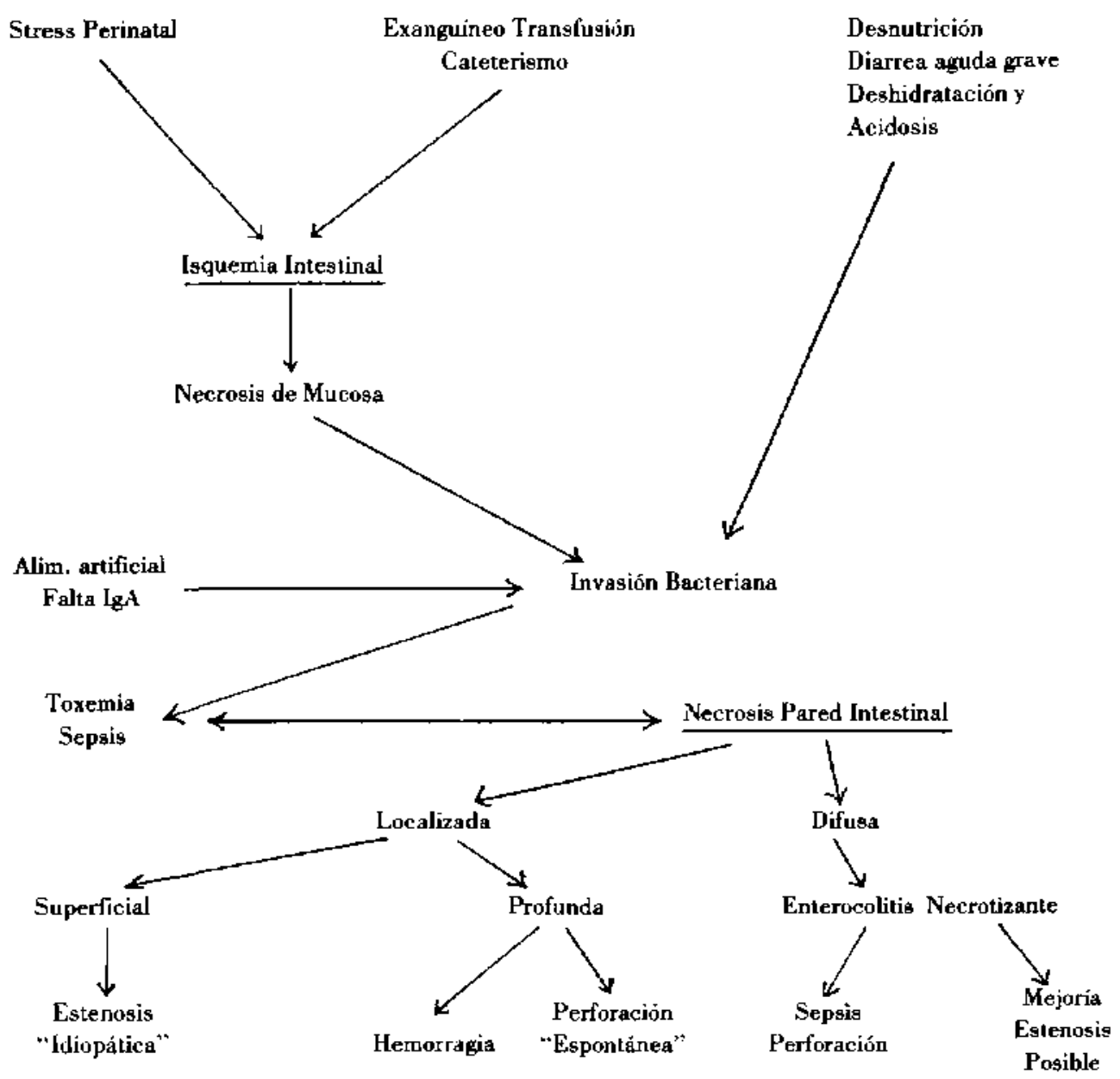

La experiencia en el Hospital Luis Calvo Mackenna desde enero de 1976 hasta junio de 1979 consiste en 27 casos que se distribuyen en la Tabla 1 .

En este mismo periodo, a partir de 1976, han sido operados 4 niños con diagnóstico de obstrucción intestinal, encontrándose en todos ellos una estenosis localizada, con componente inflamatorio, en úleon o colon. Presentamos estos 4 casos, porque, al analizarlos retrospectivamente, se puede asumir, en forma bastante razonable, que tienen relación con episodios anteriores de probable E.N.

\section{CASO 1:}

Lactante de 45 días, con 2.700 gramos de peso, que evoluciona durante 6 días con fiebre, diarrea aguda, deshidratación y acidosis. Agravamiento progresivo. Aparece agpiración biliosa, defensa muscular y rectorragia. Henograma con desviación a la izquierda acentuada. Se opera de urgen-
Tabla N. ${ }^{0} \mathbf{I}$

Enterocolitis necronizante del R.N. 1976-79

\begin{tabular}{ccccc}
\hline & \multicolumn{2}{c}{$\begin{array}{c}\text { Tratamiento } \\
\text { Médico } \\
\text { Vivos }\end{array}$} & \multicolumn{2}{c}{$\begin{array}{c}\text { Tratamiento } \\
\text { Quirúrgico }\end{array}$} \\
Recién & & & & \\
nacidos & 4 & 6 & 3 & 3 \\
Lactantes & 5 & 2 & 2 & 2 \\
& 9 & 8 & 5 & 5
\end{tabular}

17 recibjeron tratamiento médico con 9 sobrevidas.

10 fueron operados con 5 sobrevidas.

Total: 27 casos. 
cia, encontrando legiones necróticas diseminadas a k largo de todo el intestino. Se estimó que la resección masiva era incompatible con sobrevida y se optó por ofrecerle tratamiento médico agresivo y observación. La evolución fue satisfactoria, pero a los 4 meses de edad reingresa con 2.700 gramos y cuadro de obstrucción intestinal con distensión marcada y vómitos biliasos. Se reinterviene, encontrando estenosis ileal localizada a más o menos $60 \mathrm{~cm}$ de válvula ilececal, se efectúa resección de la zona estrechada y anastomosis término-terminal. El estudio histológico se comentará con los casos siguientes, por ser similar en todos ellos.

La evolución postoperatoria fue satisfactoria y a los 15 meses de edad su peso era de 10.400 gramos.

\section{CASO 2:}

Ingresa al año de edad con desnutrición severa, con peso de 4.750 gramos. Había sido estudiada desde los 35 dias de edad por síndrome de malabsorción y diarrea, que alternaba con epísodios de vómitos y dístensión abdominal. Por ûlitimo, frente a asas distendidas visibles y de lucha, el estudio radiológico y enema baritado permitieron concluir diagnóstico de estenosis del colon transverso. En la operación se confirmó el diagnóstico: regección de zona estenosada y anastomosis término-terminal del colon. Analizado cetrospectivamente este caso se obtiene, como antecedente, que nació a las 30 semanas de gestación con ruptura prematura de membranas, su peso de nacimiento fue de $1.900 \mathrm{kgs} \mathrm{y}$ necesiló oxigeno. Se realimentó artificialmente. $A$ los 5 días de vida presenta regular estado general, poco activo, con aspiración de líquido bilioso, distensión abdominal con asas visibles. La radiografia de abdomen simple revela niveles hidroaéreos y el hemograma muestra leucocitosis de 23.200, desviación a la izquierda, con elementes juveniles y mielocitos. Se opera con diagnóstico de obstrucción intestinal y en la exploración se observa intestino delgado difusamente dilatado, el colon se mostraba normal. Treinta días después de operado, por realimentación dificultosa, se practica un tránsito intestinal. sin estudio de colon, que es informado normal. Es dado de alta comenzando a efectuarse esturtio de mala absorción en otros centros, hasta que al año de edad se reopera.

Actualmente tiene 4 años y el dearrollo pondoestalural y peicomotor es normal.
CASO $3:$

Peso de nacimiento 1.640 gramos, Apgar 5, hipogdicemia $6 \mathrm{mg}$ y bilirrubinemia total $11 \mathrm{mg}$. A los 3 dias, lesiones equimóticas en extremidades y pared abdominal. Se observa poco activo y presenta periodos de apnea y cianosis. Presentó melena en varias oportunidades. El hemograma mostraba 3.600 leucocitos, 22 baciliformes y 18.000 plaquetas. Se recuperó con tratamiento médico.

A los 47 dias comienza con distensión abdominal. rechazo de alimentación y ruidos hidroaéreos aumentados. La radiografía de abdomen es comparible con obstrucción intestinal baja. Se interviene, encontrando estenosis del colon descenderte con asas intestinales adheridas. Se efectúa colostomía terminal del transverso. resecando la zona esten 6 tica. A los 9 días de operado. la colostomía está de mal aspecto, con secreción purulenta. Luego se produce evisceración, agravamiento progresivo y muerte.

\section{CASO 4:}

Peso de nacimiento 1.260 gramos, gemela de 30 semanas de geslación; nace con distress respiratorio, que es tralado con oxígeno por 4 días. Cateterismo umbilical en dos oportunidades. Agravamiento progresivo con perídos de apnea. Luego aparece distensión abdominal y vómitos biliosos. La radiografía muestra moderada distensión de asas sin neumatosis. Presenta melena en dos oportunidades. Hemograma con 32.000 plaquetas y marcada desviación a izquierda. Se recupera lentamente con tratamiento médico. hasta que cuarenta días después presenta vómitos biliosos y distensión abdominal. Weber +++ repetidus. El estudio radiológico concluye obstrucción del intestino delgado distal. Se interviene, encontranda estenosis tocalizada a $20 \mathrm{~cm}$. de válrula ileocecal con cambjo de calibre importante. Se efectúa anastomusis términu-terminal, resecando la zona estrecha.

Evolución postoperaturia satisfactoria.

\section{ESTUDIO HISTOLOGICO}

En los 4 casos existe estenosis marcada, úlcera de la mucosa, compromiso de diversas capas con infiltrado inflamatorio agudo y crónico y proliferación de tejido colágeno variable.

\begin{tabular}{|c|c|c|c|c|}
\hline & ESTENOSIS & $\begin{array}{l}\text { ULCERA } \\
\text { MUCOSA }\end{array}$ & $\begin{array}{l}\text { ALTERACION } \\
\text { OTRAS CAPAS }\end{array}$ & INFILTRADO \\
\hline CASO 1 & Lumen $2 \mathrm{~mm}$. & Sí & $\begin{array}{l}\text { Proliferación de } \\
\text { tejido colágeno. }\end{array}$ & $\begin{array}{l}\text { Linfocitario } \\
\text { plasmocelular. }\end{array}$ \\
\hline CASO 2 & Estenosis marcada & Sí & $\begin{array}{l}\text { Nódulos } \\
\text { Granulomatosos. } \\
\text { Células } \\
\text { Gigantes }\end{array}$ & $\begin{array}{l}\text { Linfocitario } \\
\text { Plasmocelular } \\
\text { Eosinófilos } \\
\text { Células } \\
\text { Gigantes }\end{array}$ \\
\hline
\end{tabular}




\begin{tabular}{|c|c|c|c|c|}
\hline & ESTENOSIS & $\begin{array}{l}\text { ULCERA } \\
\text { MUCOSA }\end{array}$ & $\begin{array}{l}\text { ALTERACION } \\
\text { OTRAS CAPAS }\end{array}$ & INFILTRADO \\
\hline CASO 3 & Lumen $8 \mathrm{~mm}$. & Sí & $\begin{array}{l}\text { Proliferación de } \\
\text { tejido colágeno. }\end{array}$ & $\begin{array}{l}\text { Submucoso y } \\
\text { Adventicial } \\
\text { Intenso. }\end{array}$ \\
\hline CASO 4 & Estenosis marcada & $\mathrm{si}$ & $\begin{array}{l}\text { Prolongación de } \\
\text { la muscular de } \\
\text { la mucosa } \\
\text { Criptas profindas }\end{array}$ & $\begin{array}{l}\text { Mononuclear } \\
\text { Submucoso } \\
\text { Intenso }\end{array}$ \\
\hline
\end{tabular}

\section{ANALISIS Y COMENTARIOS}

\section{CLADRO RESUMEN}

\begin{tabular}{|c|c|c|c|c|c|}
\hline CASO & $\begin{array}{l}\text { PESO } \\
\text { gramos }\end{array}$ & $\begin{array}{l}\text { EDAD } \\
\text { días }\end{array}$ & SINTOMAS E,Y. & $\begin{array}{c}\text { TJEMPO COMIEN- } \\
\text { ZO OBSTRUCCION } \\
\text { semanas }\end{array}$ & 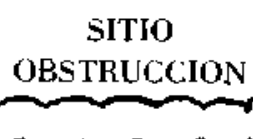 \\
\hline 1 & 2.900 & 45 & $\begin{array}{l}\text { Diarrea aguda } \\
\text { Acidosis } \\
\text { Sepsia } \\
\text { Vómitos defensa } \\
\text { Rectorragia }\end{array}$ & 10 & \\
\hline 2 & 1.900 & 5 & $\begin{array}{l}\text { Sepsis } \\
\text { Vómitos } \\
\text { Distensión abdom. } \\
\text { Rx. Obst. }\end{array}$ & 4 & \\
\hline 3 & 1.640 & 3 & $\begin{array}{l}\text { Anoxia } \\
\text { Hipoglicemia } \\
\text { Sepsis } \\
\text { Plaquetopenia } \\
\text { Distensión abdom. } \\
\text { Melena }\end{array}$ & 7 & \\
\hline 4 & 1.260 & 4 & $\begin{array}{l}\text { Stress Perinatal } \\
\text { Cateterismo umbil. } \\
\text { Sepsis } \\
\text { Vómitos } \\
\text { Distensión abdom. } \\
\text { Melena }\end{array}$ & 6 & \\
\hline
\end{tabular}

El tiempo que demoró en presentarse la sintomatología de la estenosis yarió de 4 a 10 semanas. Otros autores manifiestan que puede ser entre las 3 y 12 semanas. Joshir ${ }^{35}$ et al. llaman la atención sobre la capacidad de cicatrización en la E.N. "La epiteliza- ción puede ocurrir ya a los 3 días y el tejido de granulación y proliferación fibrolástica a los 8 días." $\mathrm{La}$ cicatrización completa puede levar a fibrosis y estenosis localizada.

$\mathrm{Al}$ analizar nuestros casos vemos que, en el pri- 
mero de ellos, la enterocolitis necronizante fue diagnosticada en el acto quinirgico y la estenosis se manifestó a los 71 días. En los otros, si bien el diagnóstico no fue afectado en la etapa aguda, los antecedentes previos, tales como stress perinatal, cateterismo umbilical, hipoglicenia, sepsis, hemograma alterado con leucocitosis, desviación a la izquierda, vómitos biliosos, defensa museular y rectorragia, que presentaron en mayor o menor grado, permiten sospechar una enterocolitis netronizante.

En el segundo caso hubo un largo periodo, 11 meses, en que, además de cuadros de subobstrucción intestinal, predominó el síndrome de malabsorción por el cual fue estudiado exhaustivamente. Al año de edad, con 4 kilos de peso, una radiografía de abdomen simple reveló gran distensión de asas intestinales y un enema baritado mostró estrechez del colon transverso. Al respecto, Lloyd, en 1973, establece que "el sindrome de malabsorción, consecutivo a daño permanente del intestino, es una complicación probable que aún no ha sido comunicada". Creemos que este caso podría responder a este enunciado.

- En los casos diagnosticados de E.N. es preciso un control alejado para descartar una estenosis u otra complicación tardía.

- Si en una intervención se encuentra una estenosis localizada, con componente inflamaturio o cicatrizal, debe investigarse si existe algún episodio previo compatible con E.N. que pueda explicar la etiología de este tipur de estenosis. Esto mismo ya en 1970 fue enunciado por Krasna, (b) quien es el primero que describe esta secuela como relacionada con episodio de enterocolitis neonatal.

- La estenosis congénita no presenta componente inflamatorio, ya que en la vida intrauterina el contenido intestinal es aséptico.

- En la estenosis postenterocolitis el estudio histológico muestra ulceración de la mucosa y alteración de las otras capas con infiltrado inflamatorio y proliferación de colágeno.

- En el sindrome de malabsorción el estudio radiológico debe ser un examen de rutina, sobre todo si existe el antecedente clínjco de asa intestinal dilatada o de lucha.

- Por último, creemos que esta secuela es pro- bable que aparezca con mayor frecuencia, ya que existe aumento de la sobrevida de prematuros y de R.N. de alto riesgo y, por lo tanto, con mayor posibilidad de enterocolitis necronizante.

\section{RESUMEN}

Se presentan 4 lactantes con cuadros de obstrucción intestinal atribuibles a enterocolitis necronizante del período de R.N.

Entre 1976 y 1979 se han atendido en el Hospital Luis Calyo Mackenna 27 casos de E.N. del R.N., 17 con tratamiento médico, 8 fallecídos y 10 operados, con 5 fallecidos.

\section{REFERENCIAS}

1 Hartow, B,; Santuli, T. J.; Heird, W. C., y cols. "An experimental study of acute neountal enteroculin - the importance of breas! milk“, J. Ped. Surg. 9: 587, 1974.

2 Burrington, $J$. "Enteroculitis necrusante dei neunato". Clinics in Perinatology $5: 1 j: 2 y, 1978$.

3 Harun, A, y cols, "Einterox ulitis necrunizanle". Rev. Chilena Pediatría 48 (2): 69, 1972 .

4 Jewett, T., y Lebenthal, E. "Acute necrotizing enteroculitis". Cutrent Prublems in Pediatrics 9 (2): 4, 1978.

${ }^{5}$ Joshi, V. V.; Winston, J. E., and Kay, S. "Nernatal necrotizing enterocolitis". Am. J. 1) 1s. Child, 126: 113, 1973.

6 Krasua, 1. H.; Becker, J. M.; Schneider, K. M., y Beck, A "Colunic Stenusis following necrutizing enteruculitis of the newborn'. J. Ped. Surc. 5 (2): 200, 1970.

7 Krasna, I. H, Fox, H. A.; Schneider, K. H., y cols. "Low molecular wcight dextrun in the treatment of necrotizing enterocalitis and midgut valvolus in intants", J. Ped. Surg. $4: 615,1973$.

" Loyd, $\boldsymbol{J}$. $R$. "The etiolugy of gustruinlestınal pertorations in the newburn". J. Ped. Surg. 4: 77, 1969.

${ }^{4}$ O'Neill, J. A,; Stuhlman, M. T, y Meng, H. C. "Necrotizing enterocolitis in the ne wbom: operalive indications". Ann. Surg. 182: $274,1975$.

10 Santulli, T. $V_{, ;}$Schullinger, J. N., Heird, W. C., y cols." Acute necrutizing enteruculitis in infant: a revie w' of 64 cases". Pediatrics 55 : $376,1975$.

11 Touloukiun, R.J.; Berdon, W. C.; Amoury. H. A., y cols, "Surgical experitence with necrotizing enterocolitis in the intant". J. Ped. Surg. 2: $38 \%, 1967$.

12 Touloukiun, R. J.; Pesch.J.N., y Spencer, R. - The pathogenesis ol is hemic gustruenteroculitis of the neonate: selective gut mucusal ischernia in asphyxiated neonatal piglets". J. Ped. Surg. 7 : $194,1972$.

13 Touloukian, $R . f$. "Neonatal necrotizing enterecolitis; An update un etiolugy, diagnosis, and treaument" , Surg. Cili. N.A. 56: $281,1976$.

it Waldhutsen, J. A.; Herendeen, $\mathrm{T}$, y King, H. "Vecrutizing enterveotitis-common cause of perforation of the colon". Surg. 54: 365,1963 . 